\title{
Exact resolution method for general 1D polynomial Schrödinger equation
}

\author{
André Voros \\ CEA-Saclay, Service de Physique Théorique \\ F-91191 Gif-sur-Yvette CEDEX (France) \\ E-mail : voros@spht.saclay.cea.fr \\ and \\ Institut de Mathématiques de Jussieu \\ CNRS UMR 7586 \\ Université Paris 7 \\ 2 place Jussieu, F-75251 Paris CEDEX 05 (France)
}

June 14, 2018

\begin{abstract}
The stationary 1D Schrödinger equation with a polynomial potential $V(q)$ of degree $N$ is reduced to a system of exact quantization conditions of Bohr-Sommerfeld form. They arise from bilinear (Wronskian) functional relations pairing spectral determinants of $(N+2)$ generically distinct operators, all the transforms of one quantum Hamiltonian under a cyclic group of complex scalings. The determinants' zeros define $(N+2)$ semi-infinite chains of points in the complex spectral plane, and they encode the original quantum problem. Each chain can now be described by an exact quantization condition which constrains it in terms of its neighbors, resulting in closed equilibrium conditions for the global chain system; these are supplemented by the standard (Bohr-Sommerfeld) quantization conditions, which bind the infinite tail of each chain asymptotically. This reduced problem is then probed numerically for effective solvability upon test cases (mostly, symmetric quartic oscillators): we find that the iterative enforcement of all the quantization conditions generates discrete chain dynamics which appear to converge geometrically towards the correct eigenvalues/eigenfunctions. We conjecture that the exact quantization then acts by specifying reduced chain dynamics which can be stable (contractive) and thus determine the exact quantum data as their fixed point. (To date, this statement is verified only empirically and in a vicinity of purely quartic or sextic potentials $V(q)$.)
\end{abstract}


We study the 1-dimensional stationary Schrödinger equation with a real polynomial potential $V(q)$ of degree $N(>2)$ on the real axis, taken in the rescaled form

$$
-\psi^{\prime \prime}(q)+[V(q)+\lambda] \psi(q)=0, \quad V(q) \equiv+q^{N}+v_{1} q^{N-1}+v_{2} q^{N-2}+\cdots+v_{N-1} q .
$$

We write the collection of coefficients as $\vec{v} \stackrel{\text { def }}{=}\left(v_{1}, \cdots, v_{N-1}\right)$, absorbing any constant term of $V$ into the spectral parameter $\lambda$ (here the sign-reverse of the usual energy).

All standard analytical treatments of this problem lead to asymptotic results at best, i.e., to semiclassical or perturbative expansions which diverge factorially and are not even Borel-summable in general ([1] - [12] provide some directly related references). Even though sophisticated techniques can sometimes convert those results to converging numerical outputs, those pathologies signal that such approaches may be mishandling the exact analytical structure of the problem (11).

In this work we further develop an alternative and entirely exact method, which until now was specialized to quantizing the spectrum in the fully homogeneous case $\vec{v}=0$ 8, 9, 12. There, it produced selfconsistent, Bohr-Sommerfeld-like quantization formulae whose implementation (through iterative numerical schemes) apparently reconstructs the exact spectrum (convergent iterations are always observed, but this fact remain to be proved). The derivation of those results was quite indirect, via Borel transformations and quantum resurgence; this made calculations lengthy, hard to generalize, and pending on still incompletely established regularity properties of the Borel-transformed solutions (cf. [4]; thm. 1.2.1 in [1]).

The approach now to be described towards the same goal is much more direct, and immediately applicable to general (inhomogeneous) potentials. It only proceeds through a pair of auxiliary eigenvalue problems on a half-line, described in Sec.1; the spectral determinants of these problems exactly express the wave-function data at the endpoint through the identity (38), and thus they inherit a bilinear (Wronskian) functional relation, eq. (43), through an analysis closely following Sibuya [2]. Exact quantization conditions are then readily extracted for the corresponding Dirichlet/Neumann spectra, representing our analytical end result: eqs.(45) (Sec.2). As in the homogeneous case, these determine the unknown spectra only as fixed points of (explicit) iterations; the convergence of the latter is then a meaningful separate question, which here remains conjectural but is numerically probed in the remaining Secs.3-4 on several test cases. First on even potentials, with the previous spectra directly giving the eigenvalues of eq.(四) over the whole line (Sec.3). Then, because eqs.(38) also supply the wave-function data at the endpoint of the half-line, the straight variation of this endpoint yields the solution of eq.(1) itself; this allows us to also test the formalism on a (ground state) eigenfunction calculation (Sec.4).

\section{Spectral preliminaries}

\subsection{Polarized boundary conditions}

We first cast eq.(1) into two eigenvalue problems with asymmetric boundary conditions. Specifically, we restrict the problem to a half-line where $V$ is confining: $[0,+\infty)$, we 
keep the square-integrability at $+\infty$, while we put a Neumann, resp. Dirichlet, boundary condition at the finite endpoint $q=0$. Both conditions then define self-adjoint operators $\hat{H}^{+}$, resp. $\hat{H}^{-}$, which have purely discrete spectra we respectively denote $\left\{E_{2 k}\right\}$ and $\left\{E_{2 k+1}\right\}$ for $k=0,1,2, \ldots$; these admit an asymptotic expansion which is the BohrSommerfeld formula reexpanded in descending fractional powers of the energy,

$$
\sum_{\nu} \tilde{b}_{\nu} E_{k}^{\nu} \sim(k+1 / 2), \quad k \rightarrow \infty \text { in } \mathbb{N}, \quad \nu=\mu, \mu-1 / N, \mu-2 / N, \cdots
$$

$$
\text { with } \quad \mu \stackrel{\text { def }}{=} \frac{1}{2}+\frac{1}{N}, \quad \tilde{b}_{\mu} \stackrel{\text { def }}{=} \oint_{p^{2}+q^{N}=1} \frac{p \mathrm{~d} q}{2 \pi}=\frac{\pi^{-1 / 2}}{N} \Gamma\left(\frac{1}{N}\right) / \Gamma\left(\frac{3}{2}+\frac{1}{N}\right),
$$

$$
\text { and } \quad \tilde{b}_{\mu-j / N}(\vec{v})=\text { a polynomial in the } v_{j^{\prime}}\left(j^{\prime} \leq j\right), \text { e.g., } \quad \tilde{b}_{\mu-1 / N}=-\frac{2 v_{1}}{\pi N} \text {; }
$$

in complete generality, the series depends on the sector (Neumann vs Dirichlet): $\tilde{b}_{\nu}=\tilde{b}_{\nu}^{ \pm}$, but not until $\nu=-3 / 2$; whereas we will invoke those coefficients (and related ones, see Sec.1.4) only in the leading range $\{\nu>-\mu\}$, before this complication appears. (If $V$ is an even polynomial, the two spectra coincide with the even vs odd parity components of the spectral problem on the whole line, hence their asymptotics coincide to all orders.)

Concerning the exponent $\mu$ (the growth order), the forthcoming arguments often assume the property $\mu<1$ (i.e., $N>2$ ) and always $\mu \neq 1$ : the case $N=2$ (singular) must be ruled out [12].

Then, up to sign, the eigenvalues read as the zeros of the Fredholm determinants

$$
\Delta^{ \pm}(\lambda) \stackrel{\text { def }}{=} \prod_{\substack{\text { even } \\ \text { odd }}}\left(1+\lambda / E_{k}\right)
$$

these infinite products converge to entire functions of order $\mu$ in the variable $\lambda$, also entire in the parameters $\vec{v}[2]$.

At fixed $(\lambda, \vec{v})$, let $\psi_{\lambda}(q)$ denote a recessive solution of eq.(1) (i.e., a solution exponentially decreasing as $q \rightarrow+\infty$, which is unique up to normalization). Then $\psi_{\lambda}(0)$ vanishes simultaneously with $\Delta^{-}(\lambda)$, (and likewise for $\psi_{\lambda}^{\prime}(0)$ and $\Delta^{+}(\lambda)$ ). However, the relations connecting them retain cumbersome factors unless each of $\Delta$ and $\psi$ is suitably renormalized. The procedure for the determinants is the well-known zeta-regularization, but a separate rescaling of the wave functions also contributes in parallel to optimal simplification.

\subsection{The spectral (or functional) determinants}

By Mellin-transforming eq.(2), the spectral zeta functions (in analogy with [7])

$$
Z^{ \pm}(s) \stackrel{\text { def }}{=} \sum_{\substack{\text { even } \\ \text { odd }}} E_{k}^{-s} \quad\left(\text { and } \quad Z(s) \stackrel{\text { def }}{=} Z^{+}(s)+Z^{-}(s)\right) \quad(\operatorname{Re} s>\mu)
$$


are seen to extend meromorphically to lower Re $s$ values, and to be regular at integer points. An effective analytical continuation is brought by an Euler-Maclaurin summation formula which uses the asymptotic information (2): the regularized form

$$
Z^{ \pm}(s)=\lim _{K \rightarrow+\infty}\left\{\sum_{k<K} E_{k}^{-s}+\frac{1}{2} E_{K}^{-s}-\frac{1}{2} \sum_{\left\{\nu>\nu_{0}\right\}} \frac{\nu \tilde{b}_{\nu}}{(-s+\nu)} E_{K}^{-s+\nu}\right\} \quad \text { for } k, K \text { even }
$$

converges as soon as $\nu_{0}$ can be taken below $(\operatorname{Re} s)$. At the regular point $s=0$, this gives

$$
Z^{ \pm}(0)=\frac{\tilde{b}_{0}}{2} \pm \frac{1}{4}
$$

The point $s=0$ further serves to define spectral determinants, as zeta-regularized products: $\operatorname{det} \hat{H}^{ \pm} \stackrel{\text { def }}{=} \exp \left[-Z^{ \pm \prime}(0)\right]$ and, by straightforward extension,

$$
D^{ \pm}(\lambda) \stackrel{\text { def }}{=} \operatorname{det}\left(\hat{H}^{ \pm}+\lambda\right) \equiv \exp \left[-Z^{ \pm \prime}(0)\right] \Delta^{ \pm}(\lambda) \text {. }
$$

This has the even more explicit Euler-Maclaurin representation

$$
\begin{aligned}
& \log D^{ \pm}(\lambda)= \lim _{K \rightarrow+\infty}\left\{\sum_{k<K} \log \left(E_{k}+\lambda\right)+\frac{1}{2} \log \left(E_{K}+\lambda\right)\right. \\
&\left.-\frac{1}{2} \sum_{\{\nu>0\}} \tilde{b}_{\nu} E_{K}^{\nu}\left(\log E_{K}-\frac{1}{\nu}\right)\right\} \text { for } k, K \text { even } \\
& \text { odd }
\end{aligned}
$$

Crucially, we can and will form these zeta-regularized products for general sequences admitting asymptotics of the form (2), including complex spectra [14] in which case the formulae are interpreted by analytical continuation from $\{\lambda>0, \vec{v}=0\}$. The $\left\{E_{k}\right\}$ are the zeros of the zeta-regularized product and they completely specify it (as opposed to a Hadamard infinite product, for which at least a normalizing factor has to be supplied independently); this normalization commutes with global spectral translations (unlike eq.(丰)).

\subsection{Absolute WKB normalization for recessive solutions}

A recessive solution of eq.(11) on $\mathbb{R}^{+}$admits an exact WKB representation [6] (for $\lambda>$ $\left.-\inf _{\mathbb{R}^{+}} V\right)$

$$
\psi_{\lambda, q_{0}}(q) \equiv u(q)^{-1 / 2} \mathrm{e}^{-\int_{q_{0}}^{q} u\left(q^{\prime}\right) \mathrm{d} q^{\prime}} \quad \text { where, for } q \rightarrow+\infty, \quad u(q) \sim \Pi(q) \stackrel{\text { def }}{=}(V(q)+\lambda)^{1 / 2} .
$$

This normalization awkwardly depends on the base point $q_{0}$, so we seek a more intrinsic one with $q_{0}=+\infty$ :

$$
\psi_{\lambda}(q) \stackrel{\text { def }}{=} u(q)^{-1 / 2} \mathrm{e}^{\int_{q}^{+\infty} u\left(q^{\prime}\right) \mathrm{d} q^{\prime}}
$$


However, only $(u-\Pi)(q)$ is integrable at infinity whereas $u(q) \sim \Pi(q) \sim q^{N / 2}$, so we add a further prescription,

$$
\int_{q}^{+\infty} u\left(q^{\prime}\right) \mathrm{d} q^{\prime} \stackrel{\text { def }}{=} \int_{q}^{+\infty}(u-\Pi) \mathrm{d} q^{\prime}+\left.\int_{q}^{+\infty}\left(V\left(q^{\prime}\right)+\lambda\right)^{-s} \mathrm{~d} q^{\prime}\right|_{s=-1 / 2}
$$

where the latter integral is now defined by analytical continuation from the domain $\{\operatorname{Re} s>1 / N, \lambda>-\inf V\}$. For the basic example $V(q)=q^{N}$, in the notations of eq.(3),

$$
\int_{0}^{+\infty}\left(q^{N}+\lambda\right)^{-s} \mathrm{~d} q \equiv \frac{\Gamma\left(s-\frac{1}{N}\right) \Gamma\left(\frac{1}{N}\right)}{N \Gamma(s)} \lambda^{-s+1 / N} \Rightarrow \int_{0}^{+\infty}\left(q^{N}+\lambda\right)^{1 / 2} \mathrm{~d} q=\frac{\pi \tilde{b}_{\mu}}{2 \sin \pi \mu} \lambda^{\mu} .
$$

In general, however, $\int_{q}^{+\infty}(V+\lambda)^{-s}$ develops a singularity at $s=-1 / 2$, which will explicitly affect many formulae hence requires further study. This singularity is immediately seen to be independent of $q$ (since $\int_{q}^{q^{\prime \prime}}(V+\lambda)^{-s} \mathrm{~d} q^{\prime}$ is finite for $q, q^{\prime \prime}$ finite), and of $\lambda$ thanks to the regular right-hand side (at $s=-1 / 2$ ) of the functional relation

$$
\frac{\partial}{\partial \lambda} \int_{q}^{+\infty}(V+\lambda)^{-s} \equiv-s \int_{q}^{+\infty}(V+\lambda)^{-(s+1)}
$$

That singularity is therefore an intrinsic feature of the potential $V$ alone.

The desired analytical continuation of $\int_{q}^{+\infty}(V+\lambda)^{-s}$ can be performed leftwards from $\{\operatorname{Re} s>1 / N\}$ using the functional relation (14) in reverse, in complete analogy with a method exposed for spectral zeta functions in [13]. It follows therefrom that the only obstructions to regular continuation lie in the leading large- $\lambda$ behavior of $\int_{q}^{+\infty}(V+\lambda)^{-s}$, which decomposes into powers $\lambda^{-s+\rho}$, the integration of which $\left(\right.$ as $\left.\lambda^{-s+\rho+1} /(-s+\rho+1)\right)$ yields only simple-pole singularities. Consequently, a convenient regularization for the definition (12) allowing such a pole at $s=-1 / 2$ is this finite part prescription,

$$
\begin{gathered}
\int_{q}^{+\infty}(V+\lambda)^{1 / 2} \stackrel{\text { def }}{=} \lim _{s=-1 / 2}\left[\int_{q}^{+\infty}(V+\lambda)^{-s}-\frac{R}{s+1 / 2}\right] \\
\text { with } R \stackrel{\text { def }}{=} \operatorname{Res}_{s=-1 / 2} \int_{q}^{+\infty}(V+\lambda)^{-s}=R(\vec{v}), \quad \text { independent of }(q, \lambda) .
\end{gathered}
$$

The new normalization (11) is then fully defined; it commutes with spatial translations.

We can alternatively describe eq.(11) in the same framework [13] but using $q$ as integration variable instead of $\lambda$. Thus, on the basis of the expansion

$$
(V(q)+\lambda)^{1 / 2} \sim \sum_{\sigma} \beta_{\sigma} q^{\sigma} \quad \text { for } q \rightarrow+\infty, \quad\left(\sigma=\frac{N}{2}, \frac{N}{2}-\frac{1}{N}, \cdots\right),
$$

$\int_{q}^{+\infty}(V+\lambda)^{1 / 2}$ gets specified as the indefinite integral consistent with the "symbolic integration" rules

$$
\int_{q}^{+\infty}(V+\lambda)^{1 / 2} \sim \sum_{\sigma} \beta_{\sigma} \int_{q}^{+\infty} q^{\prime \sigma} \mathrm{d} q^{\prime}, \quad \int_{q}^{+\infty} q^{\prime \sigma} \mathrm{d} q^{\prime} \stackrel{\text { def }}{=}\left\{\begin{array}{cc}
-q^{\sigma+1} /(\sigma+1) & (\sigma \neq-1) \\
-\log q & (\sigma=-1)
\end{array}\right.
$$


Substitution into eq.(11) then yields the asymptotic behavior

$$
\psi_{\lambda}(q) \sim q^{-\left(N / 4+\beta_{-1}\right)} \exp \left\{-\sum_{\{\sigma>-1\}} \beta_{\sigma} q^{\sigma+1} /(\sigma+1)\right\} \quad(q \rightarrow+\infty)
$$

(with no outer constant prefactor); this shows that the solution (11) exactly reproduces the subdominant solution defined in [2] (ch.2, Sec.6).

\subsection{Residues, residues}

A digression is needed here to better understand the residue $R$ from eq.(16): by relating various asymptotic expansions we will find an identity, eq.(30), among several independently defined constants including $R$.

a) We first consider the quantum partition function of the operator $-\mathrm{d}^{2} / \mathrm{d} q^{2}+V(|q|)$ on the whole line, namely

$$
\theta(t) \stackrel{\text { def }}{=} \sum_{k=0}^{+\infty} \mathrm{e}^{-t E_{k}}
$$

This admits a $t \downarrow 0$ asymptotic expansion obtainable as in the case $V(q)=|q|^{N}$, but incorporating the lower-degree terms of $V$ as perturbations:

$$
\theta(t) \sim \sum_{\nu} \tilde{c}_{-\nu} t^{-\nu}, \quad t \downarrow 0, \quad-\nu=-\mu,-\mu+\frac{1}{N},-\mu+\frac{2}{N}, \cdots
$$

(as with eq.(2), this expansion is taken only to some finite order here). The leading nonclassical contribution to eq.(21) again arises from the $|q|^{N}$ term for which it is known to be $\mathrm{O}\left(t^{\mu}\right)$; therefore, below this order, eq.(21) also applies to (and is more easily computed from) the classical partition function, i.e.,

$$
\theta_{\text {cl }}(t)=\int_{\mathbb{R}^{2}} \frac{\mathrm{d} p \mathrm{~d} q}{2 \pi} \mathrm{e}^{-\left(p^{2}+V(|q|) t\right.} \equiv \frac{1}{\sqrt{\pi t}} \int_{0}^{+\infty} \mathrm{e}^{-V(q) t} \mathrm{~d} q .
$$

We now Mellin-transform both partition functions (20) and (22) term by term, as

$$
\begin{gathered}
\frac{1}{\Gamma(s)} \int_{0}^{+\infty} \theta(t) \mathrm{e}^{-\lambda t} t^{s-1} \mathrm{~d} t=\sum_{k=0}^{+\infty}\left(E_{k}+\lambda\right)^{-s} \stackrel{\text { def }}{=} Z(s, \lambda), \\
\frac{1}{\Gamma(s)} \int_{0}^{+\infty} \theta_{\mathrm{cl}}(t) \mathrm{e}^{-\lambda t} t^{s-1} \mathrm{~d} t=\frac{\Gamma(s-1 / 2)}{\Gamma(s) \sqrt{\pi}} \int_{0}^{+\infty}(V(q)+\lambda)^{-s+1 / 2} \mathrm{~d} q \stackrel{\text { def }}{=} Z_{\mathrm{cl}}(s, \lambda) .
\end{gathered}
$$

On the quantum side (eq.(23)), this zeta function $Z(s, \lambda)$ [13 relates to eq.(5) through $Z(s, 0) \equiv Z(s)$, and to eq. (8) through

$$
\exp [-\partial Z(s, \lambda) / \partial s]_{s=0} \equiv D(\lambda) \stackrel{\text { def }}{=} D^{+}(\lambda) D^{-}(\lambda)
$$


whereas eq.(24), drawn from the rightmost eq.(22), creates a classical picture in full analogy; in particular, the counterpart of eq.(25) evaluates using

$$
\left[-\partial Z_{\mathrm{cl}}(s, \lambda) / \partial s\right]_{s=0}=2 \int_{0}^{+\infty}(V(q)+\lambda)^{1 / 2} \mathrm{~d} q
$$

(in the sense of eq.(15)).

(The parallelism between the normalization procedures (8) for the determinants, resp. (12) for the recessive solutions, is now fully clear: they involve the same analytical continuations to $s=0$ of $Z(s, \lambda)$, resp. $Z_{\mathrm{cl}}(s, \lambda)$.)

The small- $t$ expansion (21) itself Mellin-transforms to a large- $\lambda$ expansion

$$
Z(s, \lambda) \sim \sum_{\nu} \tilde{c}_{-\nu} \frac{\Gamma(s-\nu)}{\Gamma(s)} \lambda^{-s+\nu} \quad(\lambda \rightarrow+\infty)
$$

also valid strictly above $\mathrm{O}\left(\lambda^{-s-\mu}\right)$ for the corresponding classical function (24); and the analogous treatment for the derivatives at $s=0$ [13] likewise yields

$$
\log D(\lambda) \sim \sum_{\nu} \tilde{c}_{-\nu}\left[-\Gamma(-\nu) \lambda^{\nu}\right] \quad \text { with } \quad\left[-\Gamma(-0) \lambda^{0}\right] \stackrel{\text { def }}{=} \log \lambda
$$

also valid strictly above $\mathrm{O}\left(\lambda^{-\mu}\right)$ for the analogous classical quantity (26).

Below, we will specially consider the coefficient $\tilde{c}_{0}$; by the preceding arguments it has a wholly classical origin, and it is: the coefficient of $t^{0}$ in $\theta(t)$ or $\theta_{\mathrm{cl}}(t)$; the coefficient of $\log \lambda$ in $\log D(\lambda)$ or $2 \int_{0}^{+\infty}(V(q)+\lambda)^{1 / 2} \mathrm{~d} q$; the coefficient of $\lambda^{-1}$ ("residue") in $\int_{0}^{+\infty}(V(q)+$ $\lambda)^{-1 / 2} \mathrm{~d} q$ (by setting $s=1$ in eqs.(24) and (27) for $Z_{\mathrm{cl}}$ ).

b) The zeros of the above determinant $D(\lambda)$ being $\left\{-E_{k}\right\}$, their asymptotic behavior is given by the Bohr-Sommerfeld formula (2). The following specifically holds before the latter begins to depend on the parity of $k$, which may occur for $\nu \leq-3 / 2$. Then, the two large-energy expansions (2), (28) relating to the same function have to match [6, 9]: their exponents $\{\nu\}$ must coincide, as is already manifest, and their coefficients must be related, actually as

$$
\tilde{b}_{\nu} \equiv \tilde{c}_{-\nu} / \Gamma(1-\nu) \quad(\nu>-3 / 2)
$$

This result at once describes (the leading part of) the expansion (2) and shows that it is of classical origin up to $\mathrm{O}\left(\lambda^{-\mu}\right)$ (excluded). We are then going to focus on the quantity $\tilde{b}_{0}$ which contributes a constant shift term to the semiclassical series (2).

c) In the purely classical expansion (17) of $(V(q)+\lambda)^{1 / 2}$ for $q \rightarrow+\infty$, we select the coefficient of $q^{-1}$, namely $\beta_{-1}$ (cf. [2], ch.2). By simple power counting, this coefficient is independent of $\lambda$ (for $N>2$ ) and vanishes for $N$ odd. For $N$ even, it is the residue at $q=\infty$ of the complex-analytic function $(V(q)+\lambda)^{1 / 2} \stackrel{\text { def }}{\sim} q^{(N / 2)}$ (single-valued) near $q=\infty$. We also denote it $\beta_{-1}(\vec{v})$ to stress that it is an invariant of $V$.

Our present goal is to obtain the following identifications, with $R$ as in eq. (16):

$$
Z(0) \stackrel{(i)}{=} \tilde{b}_{0} \stackrel{(i i)}{=} \tilde{c}_{0} \stackrel{(i i i)}{=}-2 R \stackrel{(i v)}{=}-\frac{2}{N} \beta_{-1}(\vec{v}) \quad(\equiv 0 \text { for } N \text { odd }) .
$$


Proof: $(i)$ immediately follows from eqs.(5,7); $(i i)$, from eq.(29) specialized at $\nu=0$; (iii), from comparing the residues at $s=0$ of the two integrals in eq.(24); finally, we prove $(i v)$ by brute force.

On the one hand, we extract $R$ from eq.(16) but with $q=0$ and integration variable rescaled by $\lambda^{-1 / N}$, as

$$
\int_{0}^{+\infty}(V+\lambda)^{-s}=\lambda^{-s+1 / N} \int_{0}^{+\infty}\left(x^{N}+1\right)^{-s}\left[1+\frac{v_{1} \lambda^{-\frac{1}{N}} x^{N-1}+\cdots+v_{N-1} \lambda^{\frac{1-N}{N}} x}{x^{N}+1}\right]^{-s} \mathrm{~d} s .
$$

The residue only affects the terms with overall weight $\lambda^{-s-1 / 2}$; when the power in brackets is expanded, the corresponding coefficient comes as

$$
\sum_{k \geq 0}(-s)(-s+1) \cdots(-s-k+1) \sum_{\left\{r_{j}\right\}} \frac{v_{1}^{r_{1}} \cdots v_{N-1}^{r_{N-1}}}{r_{1} ! \cdots r_{N-1} !} \int_{0}^{+\infty}\left(x^{N}+1\right)^{-s-k} x^{(N-1) r_{1}+\cdots+r_{N-1}} \mathrm{~d} x,
$$

the inner summation being over $(N-1)$-uples $\left\{r_{j}\right\}$ subject to $\sum_{j=1}^{N-1} r_{j}=k$ and $\sum_{j=1}^{N-1} j r_{j}=$ $1+N / 2$; hence the last written integral reduces to

$$
\int_{0}^{+\infty}\left(x^{N}+1\right)^{-s-k} x^{N k-1-N / 2} \mathrm{~d} x=\frac{\Gamma(s+1 / 2) \Gamma(k-1 / 2)}{N \Gamma(s+k)},
$$

which acquires the residue $1 / N$ at $s=-1 / 2$ for any $k$.

On the other hand, we seek $\beta_{-1}$ from the large- $q$ expansion

$$
V(q)^{1 / 2}=q^{N / 2}\left[1+\frac{v_{1}}{q}+\cdots \frac{v_{N-1}}{q^{N-1}}\right]^{1 / 2}
$$

when the power in brackets is expanded, the coefficient of $q^{-1}$ comes out as

$$
\sum_{k \geq 0}\left(\frac{1}{2}\right)\left(\frac{1}{2}+1\right) \cdots\left(\frac{1}{2}-k+1\right) \sum_{\left\{r_{j}\right\}} \frac{v_{1}^{r_{1}} \cdots v_{N-1}^{r_{N-1}}}{r_{1} ! \cdots r_{N-1} !}
$$

with exactly the same summation range as before, hence this is manifestly $N$ times the residue of eq. (32) at $s=-1 / 2$.

QED.

Finally, again by simple power counting arguments, we remark that these classical invariants are not only absent for odd $N$, but also vanish for another broad class of potentials: all purely even polynomials of degree $N$ multiple of 4 (as well as for all $N$ in the special homogeneous case: $\beta_{-1}(\overrightarrow{0}) \equiv 0$.) Barring $N=2$, nontrivial residues then first occur for non-even quartic potentials:

$$
\beta_{-1}(\vec{v})=\frac{v_{3}}{2}-\frac{v_{1} v_{2}}{4}+\frac{v_{1}^{3}}{16} \quad\left(V(q)=q^{4}+v_{1} q^{3}+v_{2} q^{2}+v_{3} q\right),
$$

and, within even potentials, for sextic ones:

$$
\beta_{-1}(\vec{v})=\frac{v_{4}}{2}-\frac{v_{2}^{2}}{8} \quad\left(V(q)=q^{6}+v_{2} q^{4}+v_{4} q^{2}\right) .
$$




\subsection{Basic identities}

Under the above notations, very simple identities connect the spectral determinants and the absolute-normalized solution:

$$
D^{-}(\lambda) \equiv \psi_{\lambda}(0), \quad D^{+}(\lambda) \equiv-\psi_{\lambda}^{\prime}(0)
$$

(The proof is an adaptation of the arguments in [6], Apps. A and D).

Next, following [2], we continue eq.(11) in the complex $q$-plane down to the rotated half-line lying in the adjacent Stokes direction, namely $\left[0, \mathrm{e}^{-\mathrm{i} \varphi / 2} \infty\right)$ where

$$
\varphi \stackrel{\text { def }}{=} \frac{4 \pi}{N+2} \quad(\text { spectral symmetry angle }) .
$$

By simple complex scaling upon $q$,

$$
\psi^{[1]} \stackrel{\text { def }}{=} \psi_{\mathrm{e}^{-\mathrm{i} \varphi} \lambda}\left(\mathrm{e}^{\mathrm{i} \varphi / 2} q ; \vec{v}^{[1]}\right)
$$

provides another solution (to eq.(四) now recessive in the Stokes direction $\mathrm{e}^{-\mathrm{i} \varphi / 2}$, where

$$
\vec{v}^{[1]} \stackrel{\text { def }}{=}\left(\mathrm{e}^{\mathrm{i} \varphi / 2} v_{1}, \mathrm{e}^{2 \mathrm{i} \varphi / 2} v_{2}, \cdots, \mathrm{e}^{(N-1) \mathrm{i} \varphi / 2} v_{N-1}\right)
$$

expresses an action of the discrete rotation group of order $(N+2)$ on the coefficients; equivalently it acts upon the potential $V$, mapping it to $V^{[1]}$, then $V^{[2]}, \cdots$ (now complex potentials). The order of the effective symmetry group is

$$
L=N+2 \text { generically, } \quad L=N / 2+1 \text { for an even polynomial } V .
$$

Now the Wronskian of the two solutions $\psi, \psi^{[1]}$ of eq.(1), a constant, can be evaluated explicitly from their respective asymptotic forms (19), both valid as $q \rightarrow+\infty$ [2], and also expressed at $q=0$ by means of the respective identities (38) for the potentials $V$ and $V^{[1]}$. Matching the two calculations then yields the fundamental bilinear identity

$$
\mathrm{e}^{+\mathrm{i} \varphi / 4} D^{+}\left(\mathrm{e}^{-\mathrm{i} \varphi} \lambda, \vec{v}^{[1]}\right) D^{-}(\lambda, \vec{v})-\mathrm{e}^{-\mathrm{i} \varphi / 4} D^{+}(\lambda, \vec{v}) D^{-}\left(\mathrm{e}^{-\mathrm{i} \varphi} \lambda, \vec{v}^{[1]}\right) \equiv 2 \mathrm{i} \mathrm{e}^{\mathrm{i} \varphi \beta_{-1}(\vec{v}) / 2} .
$$

We stress that our approach will bypass any other matching of solutions, like those required in connection problems between nonadjacent Stokes directions, which yield nontrivial Stokes multipliers...

Remarks: a) in the harmonic case $\left(V(q)=q^{2}, N=2, \varphi=\pi\right)$, many of our arguments become invalid but the end result (43) remains, except that the residue is now $\lambda$-dependent, $\beta_{-1}=\lambda / 2$ from eq.(17) (the identity (43) now verifies by applying the reflection formula for $\Gamma(z)$ to $\left.D^{+}(\lambda)=2 \sqrt{\pi} 2^{-\lambda / 2} / \Gamma\left(\frac{1+\lambda}{4}\right), D^{-}(\lambda)=\sqrt{\pi} 2^{-\lambda / 2} / \Gamma\left(\frac{3+\lambda}{4}\right)\right)$; b) identities similar to eq.(43) have also surfaced in quantum integrable theories, as "quantum Wronskian conditions" 15. 


\section{Exact quantization conditions}

For a homogeneous potential, we currently believe that the functional relation (43) and the asymptotic law (2) (imposed to some o(1) accuracy) together suffice to specify the whole spectrum exactly: indeed, we empirically recovered the spectrum as the fixed point of an (apparently) contractive mapping built using just that input [8, 9, 12]. Thus, for zetaregularized products built over sequences with prescribed Bohr-Sommerfeld asymptotics, the single Wronskian identity (43) can become so coercitive as to fix both its arguments completely. We now make the same guiding idea work for general polynomial potentials.

\subsection{The analytical result}

Guided by the homogeneous case [9], we strive to turn eq.(43) into equations where at least the Neumann $(+)$ and Dirichlet $(-)$ spectra appear decoupled. To keep, say, only the former (the other admits a mirror-image treatment), we take eq.(43) and its partner with $\vec{v}^{[-1]}$ (written invoking the homogeneity property $\beta_{-1}\left(\vec{v}^{[\ell]}\right) \equiv(-1)^{\ell} \beta_{-1}(\vec{v})$ ), then we set $\lambda=-E_{2 k}$ and eliminate $D^{-}(\lambda, \vec{v})$ from the resulting pair, to find

$$
D^{+}\left(\mathrm{e}^{-\mathrm{i} \varphi} \lambda, \vec{v}^{[1]}\right) /\left.D^{+}\left(\mathrm{e}^{+\mathrm{i} \varphi} \lambda, \vec{v}^{[-1]}\right)\right|_{\lambda=-E_{2 k}}=-\mathrm{e}^{-\mathrm{i} \varphi / 2+\mathrm{i} \varphi \beta_{-1}(\vec{v})} .
$$

Whereas the procedure for $V(q)=q^{N}$ closed upon itself at once, this general one invokes the complex-rotated potentials $V^{[ \pm 1]}$ and, step by step, all the $L$ partner potentials $V^{[\ell]}$ to reach closure. The spectra $\left\{E_{2 k}^{[\ell]}\right\}$ (mostly complex) then altogether make up the independent unknowns (they are not independent as analytical continuations of each other in complex $\vec{v}$-space, but we are unable to get explicit relations expressing this).

Next, absolute phases are specified in eq.(44) (and its cyclic permutations on the $\vec{v}^{[\ell]}$ ) by reference to the homogeneous case [9]. This operation is essential to create quantization relations in the Bohr-Sommerfeld form (governed by an explicit quantum number $k=$ $0,1,2, \ldots)$, but now exact. Our final result, in a synthetic notation, is an uncoupled pair of systems (using either (,$+ k$ even) for Neumann, or $(-, k$ odd) for Dirichlet), each built of $L$ exact quantization conditions: one per potential $V^{[\ell]}$ (with $\ell$ integer $\bmod L$ ),

$$
\begin{aligned}
& \Sigma_{ \pm}^{[\ell]}\left(E_{k}^{[\ell]}\right)=\pi\left[k+\frac{1}{2} \pm \frac{N-2}{2(N+2)}\right]+(-1)^{\ell} \varphi \beta_{-1}(\vec{v}) \quad \text { for } k={ }_{1,3,5, \ldots}^{0,4, \ldots} \\
& \text { where } \quad \Sigma_{ \pm}^{[\ell]}(E) \stackrel{\text { def }}{=}-\mathrm{i}\left[\log D^{ \pm}\left(-\mathrm{e}^{-\mathrm{i} \varphi} E, \vec{v}^{[\ell+1]}\right)-\log D^{ \pm}\left(-\mathrm{e}^{+\mathrm{i} \varphi} E, \vec{v}^{[\ell-1]}\right)\right]
\end{aligned}
$$

the branches of $\log D^{ \pm}(\lambda)$ being taken by continuity from $(\lambda=0, \vec{v}=0)$.

The harmonic case $V(q)=q^{2}$ (with $N=L=2, \varphi=\pi$ ) holds again with a degenerate structure, $\Sigma_{ \pm}^{[\ell]}(E) \equiv 0$ but $\beta_{-1}=-E / 2$.

\subsection{Discussion}

All following considerations are meant in either spectral sector independently (Neumann, resp. Dirichlet), with all quantum numbers accordingly kept even, resp. odd. 
Eqs. (45) form a system of constraints tying each $E_{k}^{[\ell]}$ at fixed $\ell$ to the two other spectra $\left\{E_{j}^{[\ell-1]}\right\},\left\{E_{j}^{[\ell+1]}\right\}$ (whose zeta-regularized products build the determinants defining $\Sigma_{ \pm}^{[\ell]}$ ). The system of all such points in interaction is better displayed, in proper relative positions, if each spectrum is suitably rotated (we then call it a 'chain', as a more general name): for $\ell=0, \ldots, L-1(\bmod L)$, the $\ell^{\text {th }}$ such chain is the set $\left\{\mathrm{e}^{\mathrm{i} \ell \varphi} E_{k}^{[\ell]}\right\}$, and each of its points is under the influence of every point in the two adjacent $\left((\ell \pm 1)^{\text {th }}\right)$ chains - through the logarithm of their complex difference which enters eq.(15) via the formula (9). (In the generic case $L=N+2$, adjacency is correctly shown on a double covering of the circle of asymptotic directions; the circle itself suffices only in the purely even case $L=N / 2+1$.)

The $\ell^{\text {th }}$ equation is now a complex one precisely when its unknowns $E_{k}^{[\ell]}$ are themselves complex, i.e., for $\ell \neq 0$ or $L / 2$ : eq. (45) thus remains a formally 'complete' system of mutual constraints for the unknowns $E_{k}^{[\ell]}$. As in the homogeneous case we then surmise that eqs.(45) are not only exact, but also genuinely complete, quantization conditions; i.e., they have the capacity to determine all their unknowns provided the asymptotic condition (22) is also enforced upon each spectrum $\left\{E_{k}^{[\ell]}\right\}$ separately (using the rotated coefficients $\left.\vec{v}^{[\ell]}\right)$. If moreover this resolution can be performed in any constructive way, then we may argue that the analytical formulae (45) themselves are "giving" the solution of the stated spectral problem (Dirichlet or Neumann on the half-line).

We will now report numerical experiments, mostly upon quartic potentials, which empirically confirm our conjecture in some regions of parameter space, by achieving effective computations of the spectra out of eqs.(45) (plus eqs.(2)).

\section{Numerical tests of exact spectrum quantization}

At present we can further resolve eqs.(145) only numerically. We will essentially seek to solve them by successive approximations for each level $E_{k}^{[\ell]}$ in turn (by Newton's rootsearching method), but stopping at some finite $k_{\max }$, beyond which all eigenvalues can assume semiclassical values instead, once for all. The height of the cutoff $k_{\max }$, together with the depth in use for the semiclassical expansion (2), control the final accuracy. As for the zeta-regularized products involved, we use formula (9) (again adding higher expansion terms to improve the $k_{\max } \rightarrow \infty$ convergence).

The preceding formalism immediately provides the quantization of levels for an even polynomial potential on the real line: parity symmetry splits the spectrum into even and odd sectors, which exactly correspond to the Neumann and Dirichlet problem on the half-line, respectively. As a side effect, parity also halves the order of symmetry $L$ (cf. eq.(42)).

\subsection{Even quartic oscillators}

We now test the exact framework upon the quantization of levels for even potentials $V(q)=q^{4}+v_{2} q^{2}$ on the real line (the order of symmetry being $L=3$ ). 
To the initially real $v_{2}$ giving the real spectrum $\left\{E_{k}\right\}$ are then associated: a complex spectrum $\left\{E_{k}^{\prime}\right\}$ for the coupling constant $\mathrm{j} v_{2}$, and $\left\{E_{k}^{\prime \prime}\right\}$ for $\mathrm{j}^{2} v_{2}$ (where $\mathrm{j}=\mathrm{e}^{2 \pi \mathrm{i} / 3}$ ). The three chains $\left\{E_{k}\right\}, \mathrm{j}\left\{E_{k}^{\prime}\right\}, \mathrm{j}^{2}\left\{E_{k}^{\prime \prime}\right\}$ are shown on fig.1 for selected values of the harmonic coupling constant $v_{2}$. They altogether constitute the reduced dynamical unknowns; by reality symmetry, there are only two independent chains (one real, and one complex).

Then, in order at once to reach an equilibrium point for all constraints and to gain evidence for its uniqueness, we iteratively apply an elementary step: to recompute each chain in turn as (numerical) solution of its eq.(45) in terms of the adjacent chains at their current locations. Through an appropriate succession of such steps, we then try to build up a global process yielding a contractive iteration, as in the homogeneous case (now many more fine details can vary, and we tried but a few combinations). For us, a numerical validation of the exact quantization formulae results if one such iteration is found which displays geometric convergence to the correct spectra, i.e., a contraction ratio can be estimated with a minimum of numerical stability, and the limiting chains (checked upon their lowest five points, say) agree with independent eigenvalue calculations (e.g., matrix diagonalization).

In order to define the mandatory asymptotic behaviors of all chains, we also need reference semiclassical chains for all three rotations of $v_{2}$. These we take as the (numerical) solutions of eq.(2) pushed to 6 terms $\left(=\mathrm{O}\left(E^{-1 / 2}\right)\right.$, for better $k \rightarrow+\infty$ convergence). The same chains also conveniently serve as initial iteration data.

Such an iterative approach was found for the homogeneous case $\left(v_{2}=0\right)$ and it yielded strong contraction ratios $(\lesssim 0.4)[8,9$. However, this case also enjoyed full ternary rotation symmetry, and a qualitative change occurs now that this symmetry has to be relaxed. If we try to deform those earlier $v_{2}=0$ iterations into $v_{2}$-dependent schemes, the latter must apply synchronous updating to keep with the full ternary symmetry at $v_{2}=0$ (i.e., the chains are recomputed individually but get actually updated all at once, at each completion of a full $\ell$-cycle). Unfortunately, such iteration schemes also appear to excite new (symmetry-breaking) chain fluctuation modes possessing much lower, almost marginal, stability (contraction ratios $\approx \pm 0.9$, down to $v_{2}=0$ ). As a consequence, their convergence discontinuously drops in the immediate vicinity of $v_{2}=0$, down to unacceptably low levels. On the other hand, we empirically found that iteration schemes of a different type, which immediately update the chains one by one, can remain fairly stable in a finite neighborhood of $v_{2}=0$ (even though they are not as good as the earlier ones at the value $v_{2}=0$, where now the ternary symmetry is spontaneously broken).

The simplest such scheme ("A") consists of recomputing the real and the complex chain alternatively. For $v_{2}$ near 0 , this already appears to converge nicely and it indeed yields a first validation of the exact formalism. Still, its behavior deteriorates as $v_{2}$ grows: between $v_{2} \approx+2$ to +3 , the iterations start to converge more erratically, then our root-searching algorithm (the Newton method) goes unstable, and shortly afterwards the contraction ratios of the iteration (plotted on fig.2, left) shoot up to almost unity (for $v_{2}<2$ they were estimated both from the iteration sequence and from diagonalizing the linearized dynamics near the fixed point, and for $v_{2} \geq 2$ only the latter way). In particular, scheme "A" cannot be carried towards the harmonic limit. By contrast, it 
behaves much better in the negative direction (double-well region), showing no sign of degradation over our test range, i.e., down to $v_{2} \approx-10$ !

The positive- $v_{2}$ breakdown is possibly explained by fig.1 (right): when $v_{2}$ grows, the complex chain and its conjugate become almost degenerate towards low quantum numbers (a sort of complex tunneling effect), causing larger logarithmic pair interactions and instabilities (with the linearized-dynamics matrix entries growing like [mutual distance ${ }^{-1}$ ). An obvious remedy is then to remove the interaction between any two complex-conjugate chain points from the dynamics, by enforcing this symmetry as an a priori constraint (immediate updating also has to be kept); the so modified scheme ("B") exhibits a much more uniform linear stability indeed (fig.2, right). This scheme converges and allows to validate the exact formalism for larger values of $v_{2}$, up to $v_{2} \approx+5$. Beyond, we again become unable to get any convergence; so, global instabilities must still be creeping in, albeit more slowly. Again, the root-searching algorithm (the Newton method) diverges first, but now the linear contraction ratios themselves hardly grow at all (as obtained by diagonalization). Hence scheme "B" seems not to break down like "A" but rather to reach certain practical limits, beyond which only more elaborate implementations might resolve the case. (Perhaps, e.g., a more robust root-searching method would work, or the naive branch prescriptions implied in eq. (45) ought to be revised at such a distance from the homogeneous case.)

The preceding break-points referred to iterations in the even-parity sector. As fig.2 shows, the odd sector tends to behave more stably. A conceivably better idea might thus be to confine iterations to the odd spectrum, then to solve for the even determinants from the coupling relations (43) instead.

In conclusion, the preceding tests favor the validity and effectiveness of the exact quantization mechanism for even quartic potentials over a sizable range of the harmonic coupling $v_{2}$ about 0 , but are currently inconclusive for $v_{2} \gtrsim+5$.

In addition, we lately extended the scheme to sextic even potentials, with qualitatively similar results for the (still few) cases tested.

\subsection{Extension to non-even potentials}

In [12] we showed that the exact quantization formalism was fully valid for homogeneous potentials of any odd degree $N$, and specially for $N=1$ which has Airy functions both as solutions and as spectral determinants. The paradox in the very regular behavior of this case is that the underlying potential $V(q)=|q|$ is not even once differentiable at $q=0$. This example paved the way to the present generalization, establishing that the 'even/odd' decomposition needed in the homogeneous case was just a Neumann/Dirichlet splitting, any parity properties of the polynomial $V$ being irrelevant: an even potential over the whole line is always present as $V(|q|)$ (once its continuous differentiability is recognized as immaterial).

We thus also tested the exact quantization conditions (45) upon a few non-even quartic polynomials (now for the Dirichlet/Neumann spectra). The exact quantization conditions then involve $L=6$ distinct chains: $\ell=0,3$ are real, $(1,5),(2,4)$ being doublets of 
complex conjugates. We found one iteration sequence ("C") to converge better than others (for no clear reason): $\ell=\{0,2,3,1\}$ (then cyclically continued, with immediate updating of each chain and straight enforcement of 1-5 and 2-4 symmetry). The overall results were then comparable to those above for even polynomials (though occasionally achieving a lower numerical accuracy).

This extension of the exact quantization formalism to non-even polynomials crucially opens the way to another application (and validation) now to be described.

\section{Exact wave-function analysis}

\subsection{Analytical reconstruction of the wave function}

If no parity property is imposed upon the polynomials $V(q)$ with respect to the endpoint $q=0$, then this endpoint can be taken to an arbitrary value $a$ (on the real line, at least), thus restoring translational invariance effectively. Equivalently, 0 is kept as endpoint but the potential gets shifted to $V_{a}(q) \stackrel{\text { def }}{=} V(q+a)-V(a)$ on the half-line (becoming $V(|q|+a)-V(a)$ on the whole line).

So, we now use eq.(38) at $q=a$ instead of 0 and from right to left, to state that the absolute-normalized solution of eq.(1) at $q=a$, resp. its first derivative, are specified as

$$
\psi_{\lambda}(a) \equiv D_{a}^{-}(V(a)+\lambda), \quad \psi_{\lambda}^{\prime}(a) \equiv-D_{a}^{+}(V(a)+\lambda),
$$

in terms of the spectral determinants for the modified potential $V_{a}(q)$ on $[0,+\infty)$, denoted $D_{a}^{ \pm}$. But these in turn are canonically specified as the zeta-regularized products over their own chains of zeros; now the latter, as the eigenvalues of $\left(V_{a}\right)^{[\ell]}(q)$, are given by exact quantization conditions of the form (45), hopefully through some convergent iteration scheme as above. Thereupon, eq.(46) simply asks to apply the zeta-regularized product formula once more (at one prescribed point, $(V(a)+\lambda)$ ), in order to output the absolute-normalized solution $\psi(a)$ (or $\psi^{\prime}(a)$ ). Thus, eq.(46) acts as the last instruction in a procedure to solve the full differential equation (11) - for arbitrary $\lambda$ - through eqs.(45) (still subject to the latter giving convergent iterations, if an effective algorithm is wanted).

As a further prospect, quite general spectral problems can subsequently be tackled in principle. E.g., to find the eigenvalues $\lambda$ of eq.(11) on the whole line for a general potential

of even degree, one may proceed to solve eq.(1) as just explained but from both ends of the $q$-axis, then match the two resulting values for $\left(\psi^{\prime} / \psi\right)(a)$ at some finite location $a$.

\subsection{A numerical test}

We now validate eq.(46) with a calculation of the ground state eigenfunction for the homogeneous case $V(q)=q^{4}$. The shifted potential is then $V_{a}(q)=q^{4}+4 a q^{3}+6 a^{2} q^{2}+4 a^{3} q$, and $\psi(a)$ is the value of its determinant $D^{-}(\lambda)$ at the point $\lambda=a^{4}-E_{0}$, with the eigenvalue $E_{0} \approx 1.06036209$ being part of the input here (while it belongs to the output of an exact level calculation for $V(q)=q^{4}$ itself [8]). 
The exact quantization conditions for $V_{a}(q)$ are involving $L=6$ distinct chains (except at $a=0$, where $L=3$ by parity symmetry). We only tested calculations of $\psi(a)$ (not $\psi^{\prime}(a)$ ) for a few values of $a \geq 0$, and found the above iteration scheme "C" to converge indeed for $a \lesssim 1.7$ (with a contraction ratio per cycle $\lesssim 0.67$ ). Instabilities made us unable to pin down convergent iterations for $a \gtrsim 1.7$ (similar comments apply as for scheme "B" when $v_{2} \gtrsim+5$ ). Our output points are plotted on fig.3, against the curve produced by a standard integration routine and upon which only the global normalization was fitted (a posteriori): the results show an overall 4-5-digit agreement.

\subsection{Concluding remarks}

We have reduced the resolution of polynomial Schrödinger equations (1) to that of a discrete system of selfconsistent exact quantization conditions, eqs.(45), having as unknowns $(N+2)$ countable sequences of points subject to asymptotic boundary conditions consisting of standard Bohr-Sommerfeld formulae (因). Eqs.(45) are supplying explicit equilibrium conditions for those semi-infinite and asymptotically tethered chains.

We also have growing numerical evidence that the so reduced problem is effectively solvable in some regions of parameter space through iterations which seem to converge geometrically: it thus appears to be a self-stabilizing system, in sharp contrast with the original Schrödinger dynamics. This strengthens our following conjecture: (in such cases) the relevant equilibrium solution is realized as a fixed point of a contractive mapping, which also admits robust finite-dimensional approximations. This results in an overall indirect constructive mechanism, where exact quantization formulae explicitly specify the mapping only.

On the darker side, since eqs.(45) are very tied to the values of the degree $N$ and symmetry order $L$, they may be ill-suited to transitional regions where one of these numbers jumps (e.g, $v_{2} \rightarrow 0$ or $+\infty$ in $V(q)=q^{4}+v_{2} q^{2}$ ). Moreover, our tests still span a limited range, mostly quartic potentials close to $q^{4}$, a few even sextic ones, and (previously [9]), homogeneous potentials for their spectra only (but up to quite high degrees). Effectiveness for higher $N$, and extensions to arbitrary complex $q$ and $\vec{v}$, to more general differential systems, etc., are all conceivable but they remain open issues.

Finally we argue that, while the integration of eq.(1) may still be a remote goal using quadratures alone, another valuable question is how much the set of admissible integration methods needs to be enlarged to reach that same purpose. Our findings provide clues to the latter issue: they strongly suggest that zeta-regularized infinite products (of order $<1$ ) plus the solving of one type of fixed-point equations (which seem to have contractive and other nice properties) are pertinent additional integration tools, which are possibly sufficient to the task (already in several cases, at least).

Acknowledgment: we are grateful to R. Guida (Saclay) for letting us use his computer program to calculate hundreds of anharmonic oscillator levels with high accuracy. 


\section{References}

[1] C.M. Bender and T.T. Wu, Phys. Rev. 184 (1969) 1231-1260.

[2] Y. Sibuya, Global Theory of a Second Order Linear Ordinary Differential Operator with a Polynomial Coefficient, North-Holland, Amsterdam (1975), and refs. therein.

[3] F.W.J. Olver, Phil. Trans. Roy. Soc. London A289 (1978) 501-548, and refs. therein.

[4] J. Écalle, Cinq Applications des Fonctions Résurgentes (chap. 1), Orsay Math. preprint 84T62 (1984, unpublished).

[5] J. Zinn-Justin, Quantum field theory and critical phenomena chap. 40, Oxford Univ. Press, Oxford (1989), and refs. therein.

[6] A. Voros, Ann. Inst. H. Poincaré A 39 (1983) 211-338.

[7] A. Voros, in: Zeta functions in geometry (Proceedings, Tokyo 1990), eds. N. Kurokawa et T. Sunada, Advanced Studies in Pure Mathematics 21, Math. Soc. Japan (Kinokuniya, Tokyo, 1992), pp. 327-358.

[8] A. Voros, J. Phys. A 27 (1994) 4653-4661.

[9] A. Voros, in: Quasiclassical methods (IMA Proceedings, Minneapolis 1995), J. Rauch and B. Simon eds., IMA series vol. 95, Springer, New York (1997) 189-224.

[10] E. Delabaere, H. Dillinger and F. Pham, J. Math. Phys. 38 (1997) 6126-6184; E. Delabaere and F. Pham, Ann. Phys. 261 (1997) 180-218.

[11] E. Delabaere and F. Pham, Resurgent methods in semi-classical asymptotics, Ann. Inst. H. Poincaré (Physique Théorique) (1999, in press).

[12] A. Voros, J. Phys. A32 (1999) 1301-1311.

[13] A. Voros, Commun. Math. Phys. 110 (1987) 439-465.

[14] J.R. Quine, S.H. Heydari and R.Y. Song, Trans. Amer. Math. Soc. 338 (1993) 213231.

[15] V.V. Bazhanov, S.L. Lukyanov and A.B. Zamolodchikov, Commun. Math. Phys. 190 (1997) 247-278; P. Dorey and R. Tateo, Anharmonic oscillators, the thermodynamic Bethe Ansatz, and nonlinear integral equations, preprint ITFA 98-41 (1998), hep-th/9812211; V.V. Bazhanov, S.L. Lukyanov and A.B. Zamolodchikov, Spectral determinants for the Schrödinger equation and Q-operators of Conformal Field Theory, preprint (Dec. 1998), hep-th/9812247. 


\section{Figure captions}

Fig. 1. Interacting chains for even quartic potentials $V(q)=q^{4}+v_{2} q^{2}$, shown for odd spectral sector (i.e., odd quantum number $k$ throughout). The chains occupy their equilibrium positions $\left\{\mathrm{j}^{\ell} E_{k}^{[\ell]}\right\}$ (points marked with the corresponding $\ell$-value). The homogeneous case $v_{2}=0$ (at center) has full (ternary plus complex-conjugation) symmetry. As $v_{2} \rightarrow-\infty$, the complex chains tend to shadow the resonance spectrum $\left\{ \pm(2 k+1) \mathrm{i} \sqrt{-v_{2}}\right\}$ of the potential $-\left|v_{2}\right| q^{2}$; as $v_{2} \rightarrow+\infty$, they tend to shadow the sequence $\left\{-(2 k+1) \sqrt{v_{2}}\right\}$ $\left(\ell=1\right.$ chain for the potential $\left.v_{2} q^{2}\right)$.

Fig. 2. Linear contraction ratios for iteration schemes associated with potentials $V(q)=q^{4}+v_{2} q^{2}$ (+: even spectrum; o: odd spectrum). Left: numerical estimates for iteration scheme "A" with immediate updating (see main text); although this remark is inconclusive, we add that the numerical values of the ratios remain strictly below unity (no error estimate at all is implied in our data, but 2-3 digit stability is typically seen). Right: numerically estimated moduli of the ratios for iteration scheme "B" which further decouples conjugate-pair interactions (here, various parts of data may correspond to different eigenvalue branches, some being negative or in complex pairs).

Fig. 3. Calculations of the ground-state eigenfunction for the homogeneous quartic potential $q^{4}$. + : absolute-normalized data points obtained by iterative exact quantization (scheme "C"); in superposition, $\diamond$ : numerical estimates for the corresponding contraction ratios. Curve: computer integration of the Schrödinger equation by the NAG routine D02KEF; its rescaling factor was the only number fixed by a fit. 

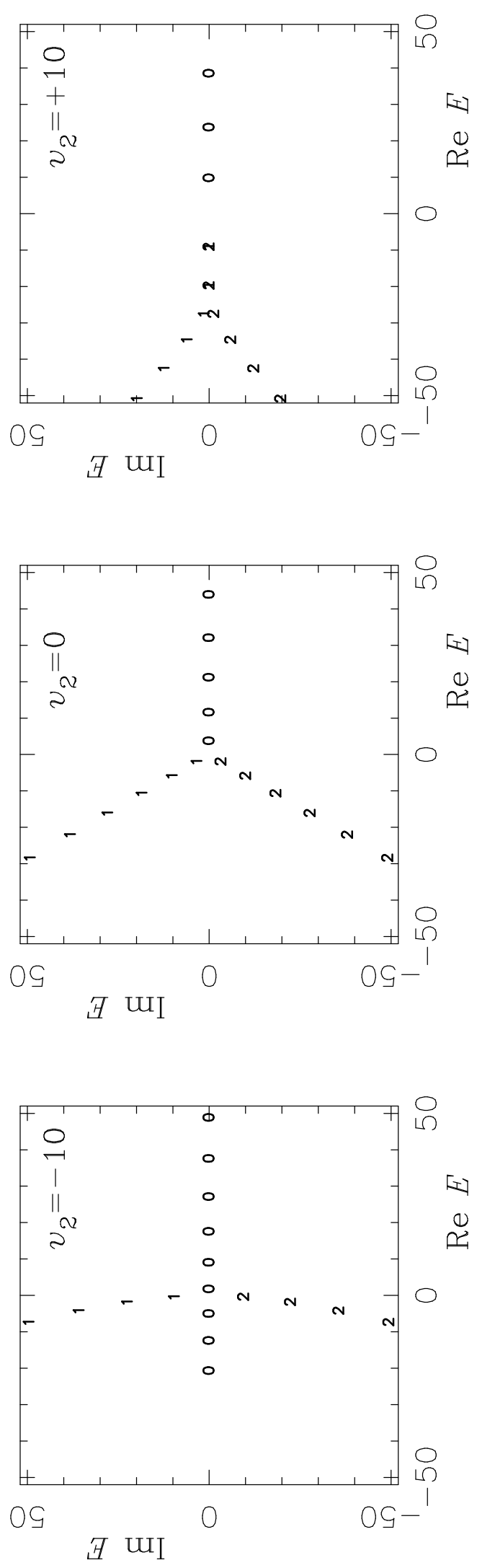
Iteration scheme "A"

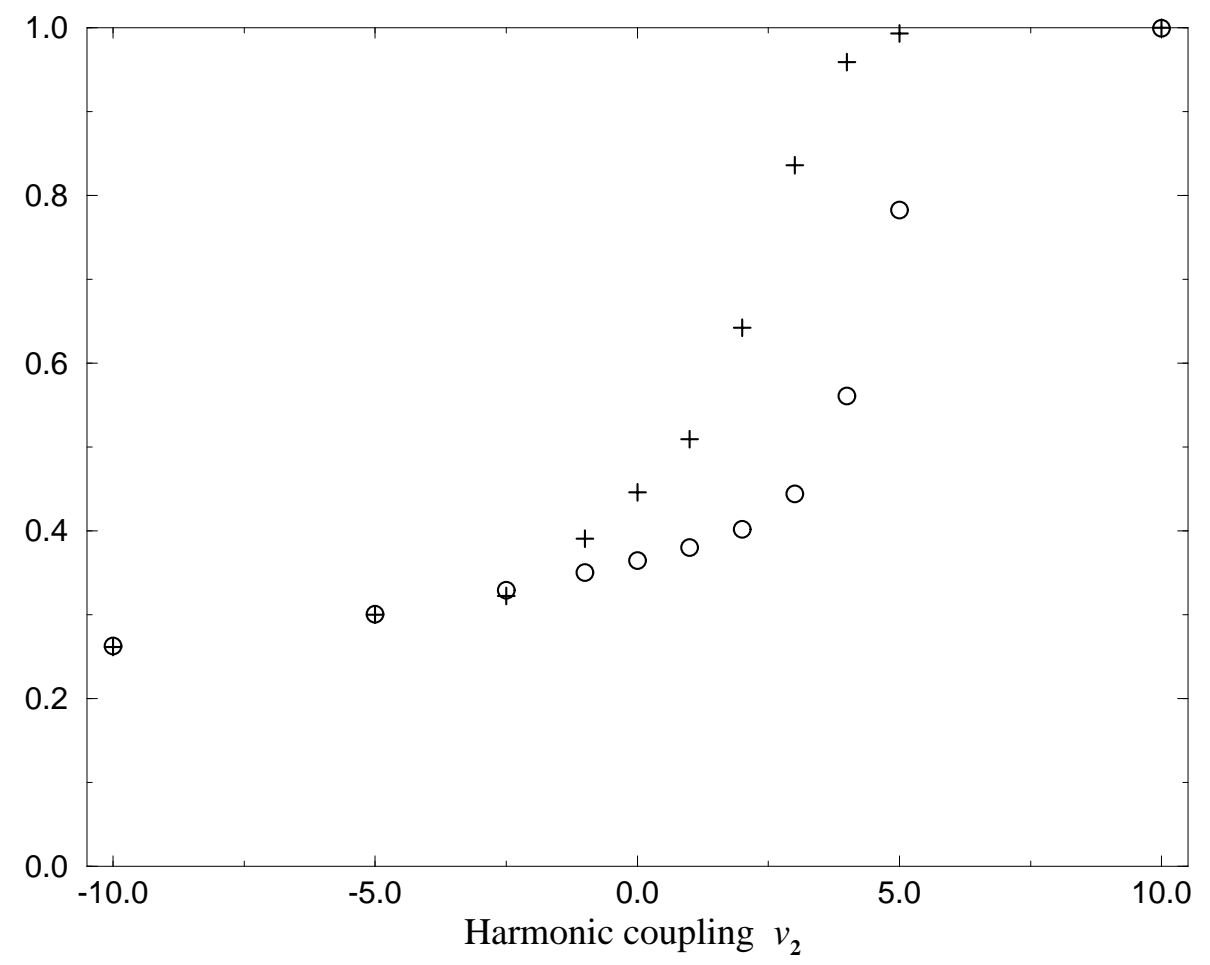

Iteration scheme "B"

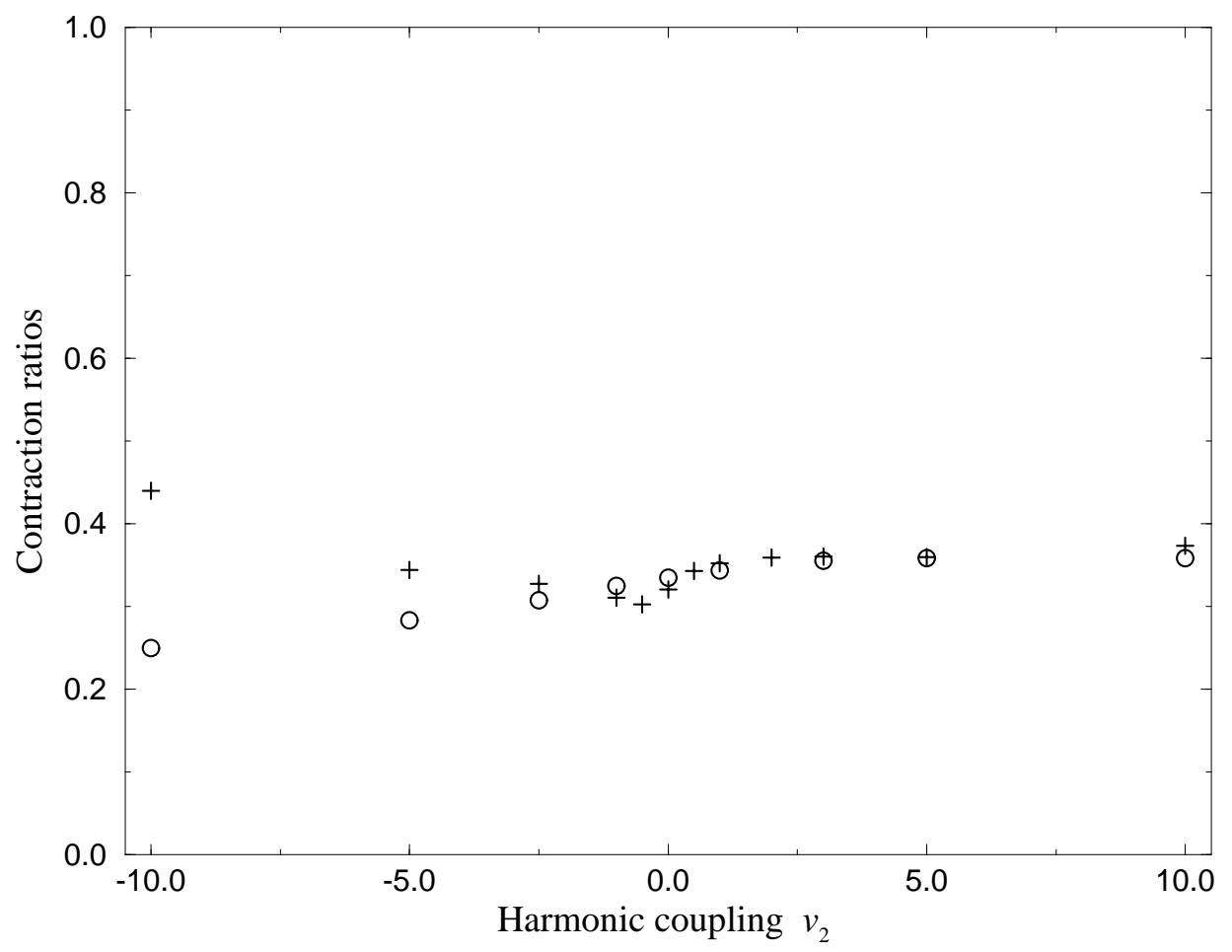




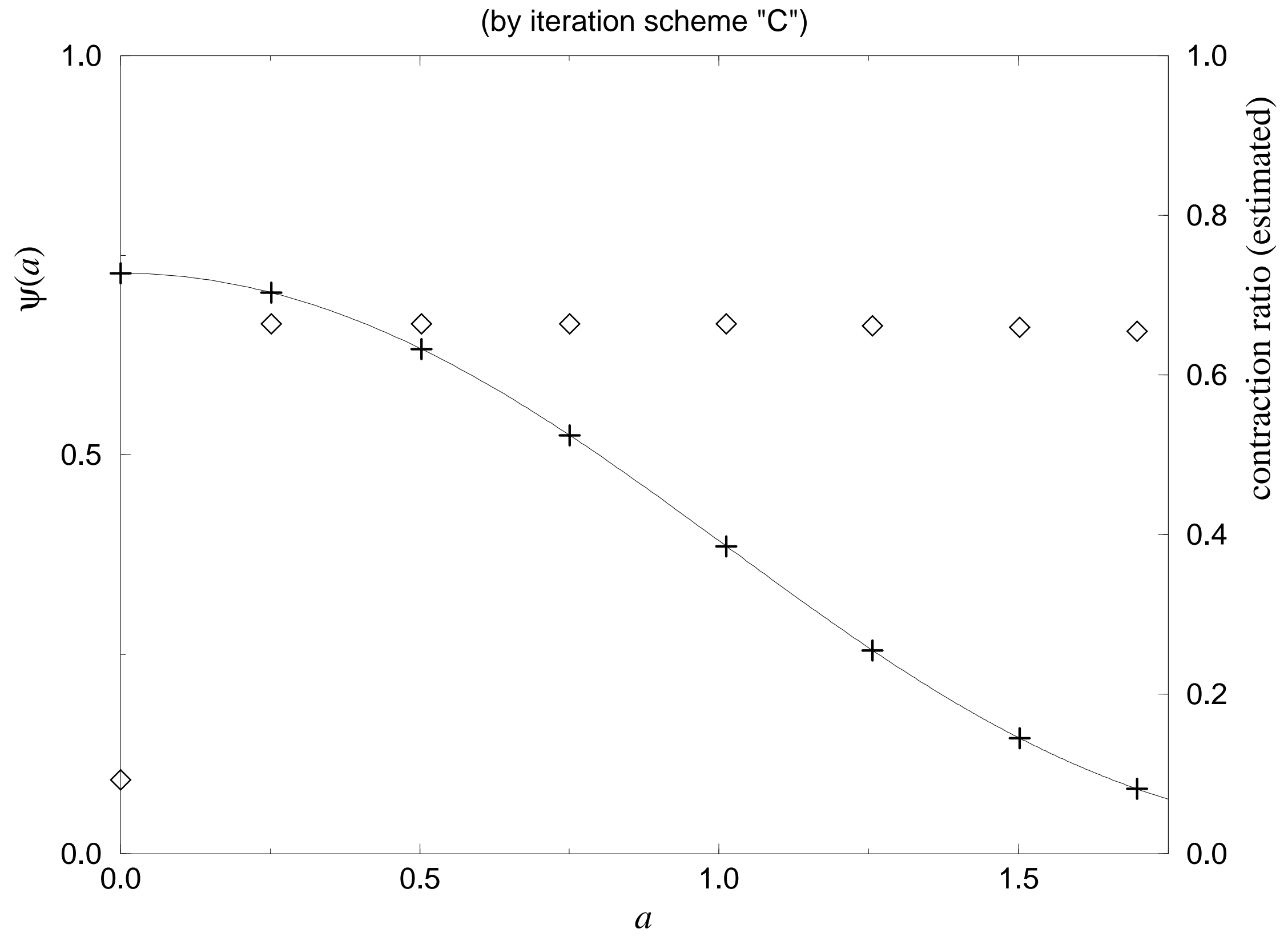

